\title{
Economic burden of major bleeding events in commercially insured patients with von Willebrand disease based on claims data from the United States
}

Mei Lu, MD, MS; Abiola Oladapo, PhD; Yanyu Wu, PhD; Sepehr Farahbakhshian, MS; and Bruce Ewenstein, MD, PhD

\section{What is already known about this subject}

- von Willebrand disease (VWD) is a rare inherited bleeding disorder resulting from a quantitative or qualitative deficit in von Willebrand factor.

- Patients with VWD, especially those with type 3 VWD, are at increased risk of major, potentially life-threatening bleeding, including bleeding of the gastrointestinal tract and central nervous system.

\author{
What this study adds \\ - Major bleeding events (mostly \\ gastrointestinal) occurred in $15 \%$ of \\ patients with VWD included in this \\ analysis of U.S. claims data. \\ - Evidence from this analysis shows that \\ patients with major bleeding events \\ incurred significantly higher health care \\ resource utilization and associated costs \\ than those without major bleeding events.
}

\author{
Author affiliations \\ Mei Lu, MD, MS, Baxalta U.S. Inc., \\ a Takeda company, Lexington, MA. \\ Abiola Oladapo, PhD; Yanyu Wu, PhD; and \\ Bruce Ewenstein, MD, PhD, Baxalta U.S. Inc., \\ a Takeda company, Cambridge, MA. \\ Sepehr Farahbakhshian, MS, Shire U.S. Inc., \\ a Takeda company, Lexington, MA.
}

AUTHOR CORRESPONDENCE: Abiola Oladapo, 617.588.8254; bladaps@yahoo.com

J Manag Care Spec Pharm. 2021;27(2):175-85

Copyright $\odot 2021$, Academy of Managed Care Pharmacy. All rights reserved.

[IRR] = 3.2; 95\% Cl=2.78-3.77), longer inpatient stays (IRR=3.9; $95 \% \mathrm{Cl}=3.12-4.93)$, and more emergency department visits (IRR $=2.0 ; 95 \%$ $\mathrm{Cl}=1.77-2.27)$ and outpatient visits (IRR $=1.3$; $95 \% \mathrm{Cl}=1.19-1.34)$ than patients without major bleeding events. Annual health care costs were significantly higher $(P<0.01)$ for patients with major bleeding events than those without them (predicted mean cost differences: total $=\$ 20,890$, pharmacy $=\$ 2,593$, and medical $=\$ 18,293)$.

CONCLUSIONS: Major bleeding events were associated with increased HCRU and costs, mostly inpatient costs. Therefore, optimizing therapy to prevent or reduce major bleeding events has the potential to reduce health care use and costs in patients with VWD. 
von Willebrand disease (VWD) is a rare inherited bleeding disorder, with a symptomatic prevalence rate of approximately 1:10,000 for patients referred to specialized tertiary care centers, or 1:1,000 for patients identified in a primary care setting., ${ }^{1,2}$ Patients with VWD have impaired hemostasis owing to a quantitative or qualitative deficit in von Willebrand factor (VWF), a plasma glycoprotein that mediates platelet adhesion and aggregation and stabilizes coagulation factor VIII in circulation. ${ }^{3,4}$

VWD is classified into 3 primary categories based on VWF phenotype to aid diagnosis, treatment, and counseling of patients with VWD., ${ }^{1,5}$ Type 1 accounts for $70 \%-80 \%$ of cases; type 2, approximately $20 \%$ of cases; and type 3 , which is the most severe form, $<5 \%$ of cases.

Most patients with VWD (70\%-80\%) present with mild to moderate bleeding symptoms (usually mucosal bleeding or bleeding after trauma or surgery), synonymous with type 1 VWD, which is the most common form. Major, potentially life-threatening bleeding can also occur, including gastrointestinal tract and central nervous system bleeding, especially in patients with type $3 \mathrm{VWD}{ }^{1,3}$

Bleeding rates associated with VWD (including serious, hard-to-treat bleeds, such as gastrointestinal bleeds) have been previously described..$^{6,7}$ Median annualized bleed rates (ABRs) before prophylaxis in the VWD International Prophylaxis Study $(\mathrm{N}=80)$ were 9.6 for menorrhagia, 9.3 for gastrointestinal bleeding, 11.9 for joint bleeding, and 11.1 for epistaxis. ${ }^{6}$ Holm et al. (2018) also reported higher rates of inpatient admissions and outpatient visits owing to bleeding events in patients with VWD than in the control population. ${ }^{7}$ These studies have not, however, reported on health care resource utilization (HCRU) and costs associated with managing these bleeding events. Management of major bleeding events would be expected to contribute substantially to the economic burden of VWD, as the majority of patients with VWD do not currently receive continuous replacement therapy.

The objective of this study was to estimate the prevalence, HCRU, and costs associated with major bleeding events among patients with VWD.

\section{Methods}

\section{PATIENT POPULATION}

In this retrospective database analysis study, patients were identified from the IBM MarketScan Commercial Claims and Encounters database and the Medicare Supplemental and Coordination of Benefits database (January 2008-December 2016) using the following eligibility criteria: $\geq 2$ diagnoses of VWD (International
Classification of Diseases, Ninth Revision, Clinical Modification $[\mathrm{ICD}-9-\mathrm{CM}]=286.4$; Tenth Revision $[\mathrm{ICD}-10-\mathrm{CM}]=\mathrm{D} 68.0$ ) recorded from different hospital admissions or outpatient visits (excluding laboratory or radiology data) and no diagnosis of acquired coagulation factor deficiency (ICD-9-CM=286.7; ICD-10-CM=D68.4) at any time. Patients with continuous health care plan enrollment for $\geq 12$ months from the eligibility start date (i.e., the date on which a patient becomes eligible for insurance benefits and thus is included in the database) were included in the prevalence estimation of major bleeding events. The observation period for occurrence of major bleeding events started at each patient's eligibility start date and ended at the end date of continuous eligibility. All data analyzed were deidentified by MarketScan and comply with the patient requirements of the Health Insurance Portability and Accountability Act.

A further 2 cohorts of patients with VWD comprising those with or without major bleeding events during the observation period were identified for the economic analysis, which compared HCRU and associated costs for patients with and without major bleeding events. The VWD cohort with major bleeding events had $\geq 1$ major bleeding event on or after the first diagnosis of VWD. The VWD cohort without major bleeding events had no major bleeding events at any time during the continuous eligibility claims period.

A major bleeding event was defined as a medical claim associated with a diagnosis code (ICD-9/10-CM; Supplementary Table 1, available in online article) for intracranial, gastrointestinal, or eye bleed in a hospital or outpatient setting or a medical claim for menorrhagia, epistaxis, or joint bleed that required a transfusion with blood products (Supplementary Table 2, available in online article) either during hospitalization or on or within 7 days after the date of diagnosis in the outpatient setting. This definition was derived from the International Society on Thrombosis and Haemostasis (ISTH) definition for major bleeding and the National Federation of Hemophilia definition for serious bleeding episodes, and it utilized a conservative approach owing to the lack of clinical information (e.g., laboratory and symptom data). The ISTH defines major bleeding in patients receiving antihemostatic medical products as bleeding in a critical area (intracranial, intraspinal, intraocular, retroperitoneal, intra-articular, pericardial, or intramuscular with compartment syndrome) and/or a bleed resulting in a fall in hemoglobin levels of $>2 \mathrm{~g} / \mathrm{dL}$ or requiring transfusion of $\geq 2$ units of whole blood or red cells. ${ }^{8}$ The National Hemophilia Foundation defines serious bleeding in patients with hemophilia as serious, life-threatening bleeds, including intracranial or spinal cord bleeds, throat bleeds, intra-abdominal bleeds, limb compartment syndromes (e.g., 
thigh, calf, forearm, upper arm), and ocular bleeds. Bleeds in all of these areas are characterized by bleeding into an enclosed space, compression of vital tissue, and potential loss of life. ${ }^{9}$

Both cohorts had $\geq 12$ months of continuous enrollment prior to the index date (i.e., the baseline period), $\geq 12$ months of continuous eligibility following the index date (i.e., the study period), and medical and pharmacy benefits in both the baseline and study periods with no capitated health care plans during the 12-month study period. For the VWD cohort with major bleeding events, the index date was defined as either the date of the first major bleeding event in the same encounter (inpatient or outpatient) as the VWD diagnosis date or the date of the first major bleeding event after the VWD diagnosis date. For the VWD cohort without major bleeding events, each patient was assigned a random index date such that the index date distribution (mean and SD) relative to the first diagnosis date of VWD matched that for the cohort with major bleeding events.

\section{PATIENT CHARACTERISTICS AND OUTCOME MEASURES}

Patient characteristics and outcome measures included patient demographic characteristics (at index date) and clinical characteristics over the baseline period, including Charlson Comorbidity Index (CCI) and comorbidities (e.g., anxiety, depression, obesity, anemia, and fatigue), overall prevalence of major bleeding events during the observation period, all-cause HCRU, and all-cause direct health care costs during the 12-month study period.

\section{MAJOR BLEEDING EVENTS}

Overall prevalence of major bleeding events was defined as the proportion of patients with VWD in the database with $\geq 1$ year of continuous eligibility who had a major bleeding event during the observation period. Annual frequency of major bleeding events was also calculated for each calendar year in the study period (i.e., 2008-2016).

\section{SENSITIVITY ANALYSIS}

As the requirement for a transfusion with blood products may have caused an underestimation in the prevalence of major bleeds, a sensitivity analysis was also conducted using an alternative definition for menorrhagia, epistaxis, and joint bleeds (i.e., a medical claim with the associated bleeding-related diagnosis code [ICD-9/10-CM codes; Supplementary Table 1] that had hemoglobin, hematocrit, or complete blood count laboratory assessments [Supplementary Table 3, available in online article] within the hospital stay or $\leq 7$ days after the diagnosis date). For intracranial, gastrointestinal, or eye bleeds, the definition used in the sensitivity analysis was the same as that in the main analysis.

\section{STATISTICAL METHODS}

All statistical analyses were performed using the Boston Health Economics Instant Health Data tool and SAS for Windows, version 9.3 (SAS Institute, Cary, NC). A 2-sided $P$ value $<0.05$ indicated statistical significance.

Patient demographics and clinical characteristics were summarized using descriptive statistics. For the economic analysis, all baseline comparisons were conducted using unadjusted analyses (i.e., 2-sample t-test or Wilcoxon ranksum test for continuous variables and a chi-square test or Fisher's exact test for categorical variables). All comparisons during the 12-month study period were conducted using adjusted analyses controlling for demographics, health plan, index year, CCI, comorbidities, thromboembolic events, and HCRU during the 12-month baseline period of continuous enrollment. A generalized linear model (GLM) with Poisson distribution, Pearson scale (to account for overdistribution of the data), and log link was used for inpatient days and number of inpatient, emergency department (ED), outpatient, or hematologist visits. A GLM with gamma distribution and log link or 2-part model was used for cost analyses; $P$ values were from the bootstrapping procedure. Health care costs were adjusted to 2016 U.S. dollars using the medical component of the U.S. Consumer Price Index.

\section{Results}

\section{PATIENTS AND BASELINE CHARACTERISTICS}

A total of 19,785 patients with VWD were identified and included in the estimation of prevalence analysis (Figure 1). The majority of patients were female $(75.2 \%)$, and the mean (SD) age was 33.6 (19.7) years (Supplementary Table 4, available in online article).

\section{PREVALENCE OF MAJOR BLEEDING EVENTS}

Of the 19,785 patients with VWD included in the prevalence analysis, 2,986 patients (15.1\%) experienced $\geq 1$ major bleeding event over a median observation period of 4 years (mean [SD] rate $=0.11$ [0.64] major bleeding events/year; Figure 2). Annual prevalence of major bleeding events ranged from 3.9\%-6.1\% between 2008 and 2016. Gastrointestinal bleeding was the most prevalent type of major bleeding event (13.4\%); the prevalence of gastrointestinal bleeds tended to increase with age (Supplementary Figure 1, available in online article). The prevalence of major bleeding events (excluding menorrhagia) was similar in both sexes. 


\section{FIGURE 1 Identification of Study Population with VWD in the IBM MarketScan Database}

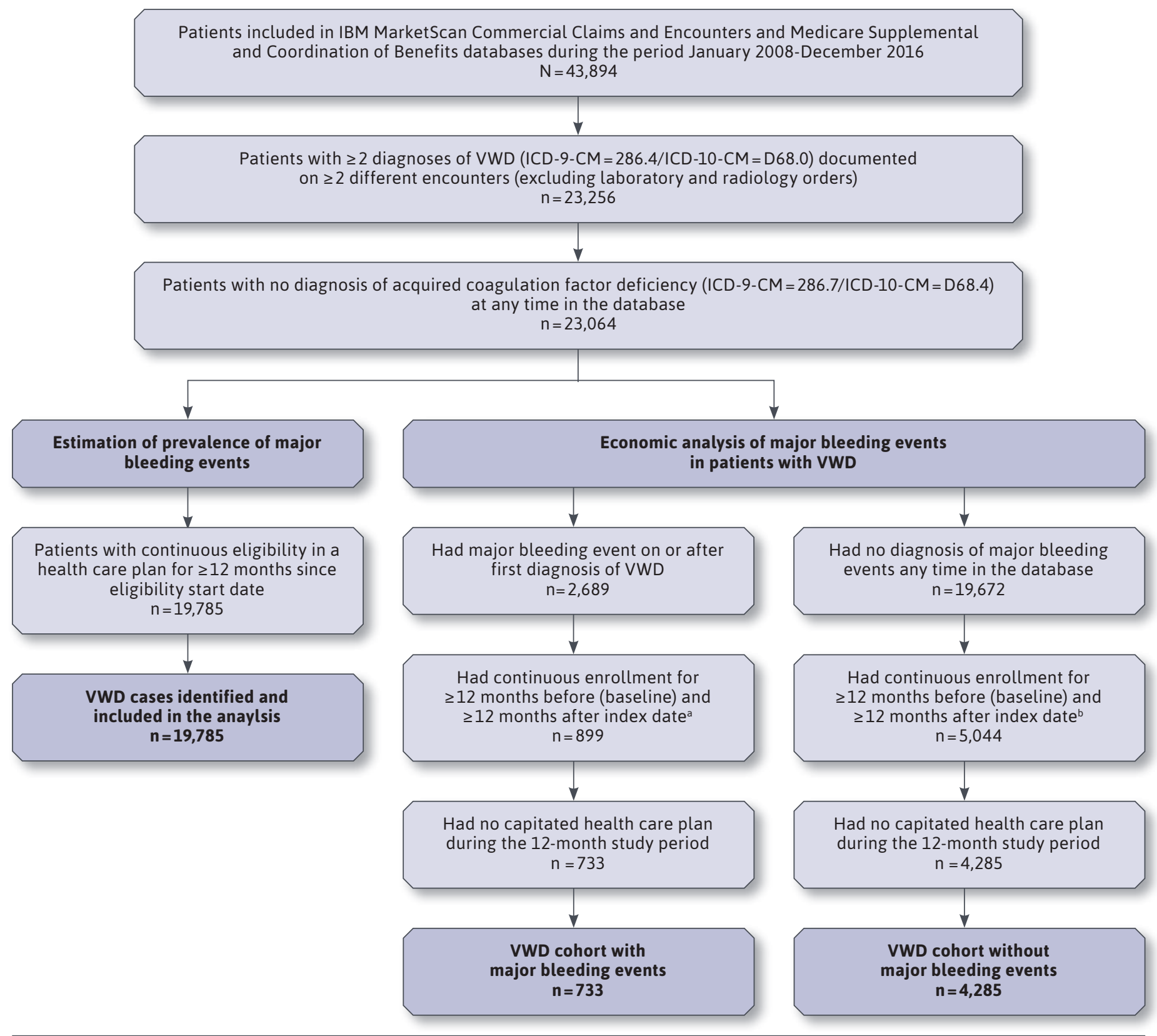

andex date was defined as the hospital admission date if the first major bleeding event was identified in a hospital or as the first diagnosis date if a major bleeding event was identified in other settings.

${ }^{b}$ Each patient was assigned an index date so that the distribution of index dates (relative to the VWD diagnosis date) matched the distribution for the cohort with major bleeding events.

ICD-9-CM = International Classification of Diseases, Ninth Revision, Clinical Modification; ICD-10-CM=International Classification of Diseases, Tenth Revision, Clinical Modification; $\mathrm{VWD}=$ von Willebrand disease 


\section{FIGURE 2 Overall Prevalence of Major Bleeding Events in Patients with VWD by Bleed Type and Sex ${ }^{\mathrm{a}}$}

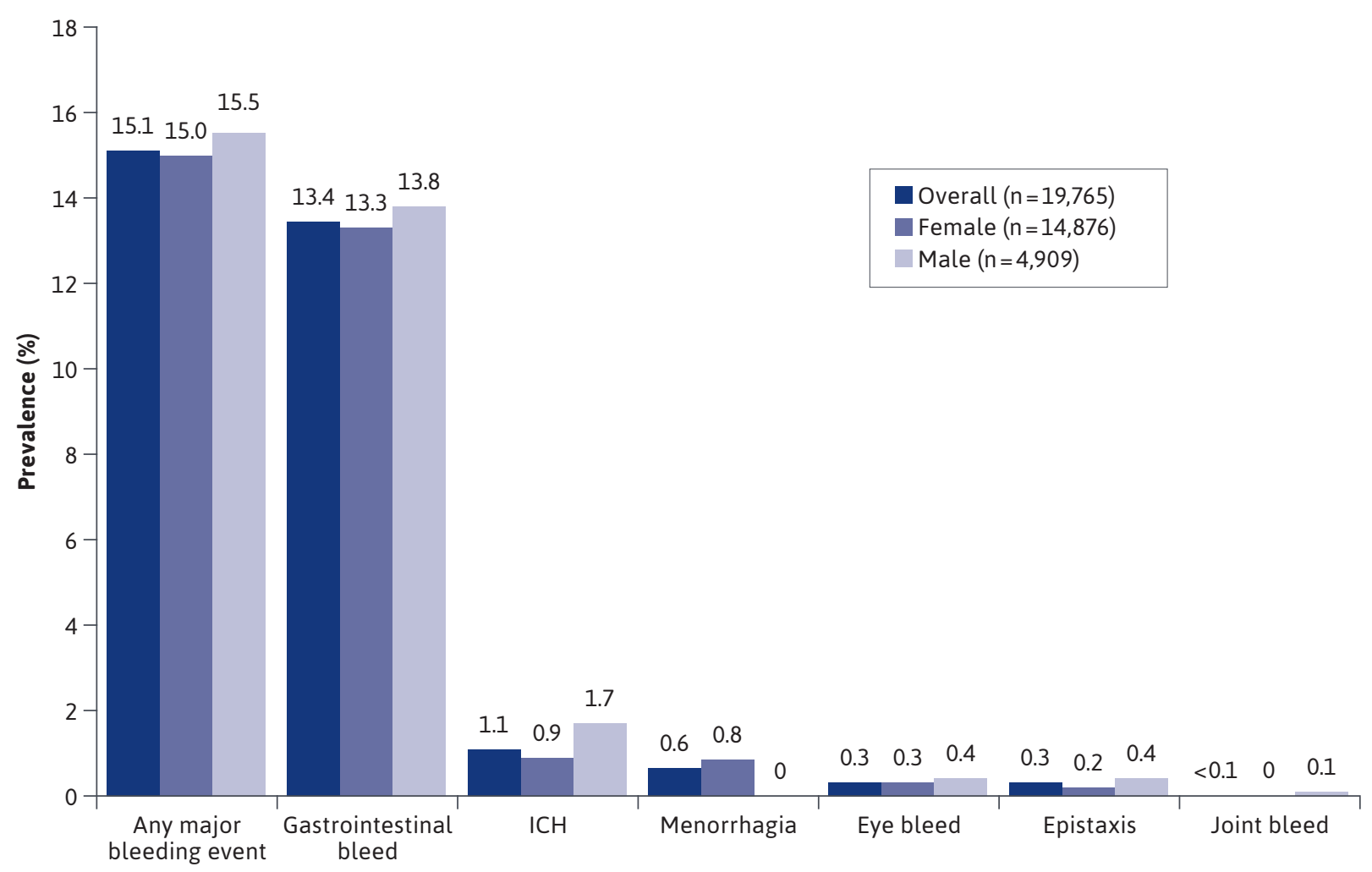

\begin{tabular}{|c|c|c|c|c|c|c|c|}
\hline Overall, n & 2,986 & 2,656 & 213 & 117 & 55 & 54 & 3 \\
\hline Female, n & 2,225 & 1,980 & 131 & 117 & 37 & 33 & 0 \\
\hline Male, n & 761 & 676 & 82 & 0 & 18 & 21 & 3 \\
\hline $\begin{array}{l}\text { Overall annual } \\
\text { frequency, mean (SD) }\end{array}$ & $0.11(064)$ & $0.09(0.44)$ & $0.01(0.45)$ & $0(0.04)$ & $0(0.06)$ & $0(0.03)$ & $0(0.01)$ \\
\hline
\end{tabular}

avalues represent numbers of patients with major bleeding events and overall prevalence (\%). Patients could have had multiple bleeding events.

bover a median 4-year follow-up period.

$\mathrm{ICH}=$ intracranial hemorrhage; $\mathrm{VWD}=$ von Willebrand disease.

Sensitivity Analysis. A higher rate of major bleeding events was observed in the sensitivity analysis than in the primary analysis (31.0\% vs. 15.1\%, respectively; SupplementaryTable 5 , available in online article). This difference was mainly driven by a higher prevalence of menorrhagia $(14.2 \%$ vs. $0.6 \%)$ in the sensitivity analysis than in the primary analysis. An increased prevalence of epistaxis (5.8\% vs. $0.3 \%)$ and joint bleeds $(0.22 \%$ vs. $0.02 \%)$ was also observed.

\section{ANALYSIS OF ECONOMIC BURDEN OF MAJOR BLEEDING EVENTS}

Of the 19,785 patients with VWD (Table 1), 5,058 patients met the criteria for the economic burden analysis, including
773 patients with $\geq 1$ major bleeding event and 4,285 patients without major bleeding events (Figure 1; Table 1). At baseline (Table 1), patients with major bleeding events were older (mean [SD] age $=44.5$ [20.1] vs. $34.2[19.5]$ years; $P<0.0001$ ) with more comorbid diseases (mean [SD] CCI score $=0.8[1.4]$ vs. $0.4[1.0] ; \mathrm{P}<0.0001)$ than those without major bleeding events, respectively. A higher proportion of patients with major bleeding events also presented with anemia, depression, fatigue, thromboembolic events $(\mathrm{P}<0.0001)$, and anxiety $(P=0.0002)$.

Health Care Resource Utilization. During the study period, patients with major bleeding events incurred significantly 


\section{TABLE 1 Baseline Characteristics of Patient Sample Included in the Economic Analysis}

\begin{tabular}{|c|c|c|}
\hline Characteristic & $\begin{array}{l}\text { Patients with } \\
\text { Major Bleeding } \\
\text { Events }(n=773)\end{array}$ & $\begin{array}{c}\text { Patients with No } \\
\text { Major Bleeding } \\
\text { Events }^{\text {a }}(n=4,285)\end{array}$ \\
\hline \multicolumn{3}{|l|}{ Age, years } \\
\hline Mean $(S D)^{b}$ & $(20.1)$ & $(19.5)^{b}$ \\
\hline Median (range) & $47 \quad(1-91)$ & $33 \quad(0-95)$ \\
\hline \multicolumn{3}{|l|}{ Age group, $\mathrm{n}(\%)^{\mathrm{b}}$} \\
\hline $0-11$ years & $34 \quad(4.4)$ & $(12.0)$ \\
\hline $12-17$ years & $50 \quad(6.5)$ & $(14.6)$ \\
\hline $18-54$ years & $431 \quad(55.8)$ & $2,333 \quad(54.4)$ \\
\hline$\geq 55$ years & $258 \quad(33.4)$ & $(19.0)$ \\
\hline \multicolumn{3}{|l|}{ Sex, n (\%) } \\
\hline Female & $583 \quad(75.4)$ & $3,191 \quad(74.5)$ \\
\hline Male & $190 \quad(24.6)$ & $1,094 \quad(25.5)$ \\
\hline \multicolumn{3}{|c|}{ Geographic region, $n(\%)$} \\
\hline Midwest & $188 \quad(24.3)$ & $1,029 \quad(24.0)$ \\
\hline Northeast & $232 \quad(30.0)$ & $1,362 \quad(31.8)$ \\
\hline South & $240 \quad(31.0)$ & $1,229 \quad(28.7)$ \\
\hline West & $104 \quad(13.5)$ & $620 \quad(14.5)$ \\
\hline Unknown & $9 \quad(1.2)$ & $(1.1)$ \\
\hline \multicolumn{3}{|l|}{ Payer, n (\%) } \\
\hline Commercial & $655 \quad(84.7)$ & $(94.0)$ \\
\hline Medicare & $118 \quad(15.3)$ & $(6.0)$ \\
\hline \multicolumn{3}{|c|}{ Health care plan, $n(\%)^{b}$} \\
\hline $\mathrm{CDHP}$ & $54 \quad(7.0)$ & $(6.9)$ \\
\hline Comprehensive & $59 \quad(7.6)$ & $(4.3)$ \\
\hline EPO & $(1.0)$ & $(1.2)$ \\
\hline $\mathrm{HDHP}$ & $(3.5)$ & $(4.8)$ \\
\hline $\mathrm{HMO}$ & 0 & 0 \\
\hline POS & $57 \quad(7.4)$ & $(7.5)$ \\
\hline POS with capitation & 0 & 0 \\
\hline PPO & $533 \quad(69.0)$ & $3,001 \quad(70.0)$ \\
\hline Unknown & $(4.5)$ & $(5.3)$ \\
\hline \multicolumn{3}{|l|}{$\mathrm{CCl}$ score } \\
\hline Mean (SD) ${ }^{\mathrm{b}}$ & $0.8 \quad(1.39)$ & $0.4 \quad(0.98)$ \\
\hline Median (range) & $0 \quad(0-10)$ & $(0-9)$ \\
\hline
\end{tabular}

continued on next page

higher HCRU than those without major bleeding events after controlling for baseline covariates (i.e., age, sex, region, health plan, index year, CCI, comorbidity [anemia, anxiety, depression, fatigue, and obesity], any thromboembolic event [as defined in Table 1], and prior medication for VWD and HCRU [inpatient, ED, and outpatient]). Patients with major bleeding events were also significantly more likely to have an inpatient admission (odds ratio $[\mathrm{OR}]=4.1 ; 95 \% \mathrm{CI}=3.41-4.97$; $\mathrm{P}<0.0001)$, ED visit $(\mathrm{OR}=1.8 ; 95 \% \mathrm{CI}=1.48-2.10 ; \mathrm{P}<0.0001)$, outpatient visit $(\mathrm{OR}=4.9 ; 95 \% \mathrm{CI}=1.79-13.43 ; \mathrm{P}=0.002)$, and a hematologist visit $(\mathrm{OR}=1.7 ; 95 \% \mathrm{CI}=1.37-2.13 ; \mathrm{P}<0.0001)$ compared with those without major bleeding events (Figure 3). Similarly, patients with major bleeding events had significantly more inpatient admissions (incidence rate ratio $[\mathrm{IRR}]=3.2 ; 95 \% \mathrm{CI}=2.78-3.77)$ and longer inpatient stays $(4.26$ days vs. 0.74 days; $I R R=3.9 ; 95 \% \mathrm{CI}=3.12-4.93)$, as well as more $\mathrm{ED}$ visits $(\mathrm{IRR}=2.0 ; 95 \% \mathrm{CI}=1.77-2.27)$ and outpatient visits (IRR=1.3; 95\% CI=1.19-1.34) than those without major bleeding events (Supplementary Figure 2; $\mathrm{P}<0.0001$ for all comparisons).

Health Care Costs. During the study period, patients with major bleeding events incurred significantly higher all-cause direct health care costs $(P<0.01)$ than patients without major bleeding events after controlling for baseline covariates (i.e., age, sex, region, health plan, index year, CCI, comorbidity [anemia, anxiety, depression, fatigue, and obesity], any thromboembolic event, prior medication for VWD, and HCRU [inpatient, ED, and outpatient]; Figure 4). The predicted mean annual total cost difference between the cohorts with or without major bleeding was \$20,890 (95\% $\mathrm{CI}=\$ 15,524-\$ 29,254)$. Pharmacy and medical costs were significantly higher for patients with major bleeding compared with those without major bleeding, with predicted mean annual cost differences of $\$ 2,593 \quad(95 \% \mathrm{CI}=\$ 910-\$ 4,873$; $\mathrm{P}=0.002)$ and $\$ 18,393(95 \% \mathrm{CI}=\$ 12,304-\$ 25,048 ; \mathrm{P}<0.01)$, respectively. Inpatient costs (mean $\pm \mathrm{SD}$ ) were also significantly higher for patients with major bleeding events than for those without major bleeding events, with a predicted mean annual cost difference of $\$ 11,595(95 \% \mathrm{CI}=\$ 6,914-$ $\$ 16,595 ; \mathrm{P}<0.01)$.

\section{Discussion}

In this large retrospective analysis of data from a U.S. commercial health care plan, approximately $15 \%$ of patients with VWD experienced a major bleeding event, most frequently gastrointestinal bleeds, over a median follow-up period of 4 years. The analysis also demonstrated significantly higher HCRU and associated costs in patients with major bleeding events than in those without. 


\section{TABLE 1}

Baseline Characteristics of Patient Sample Included in the Economic Analysis (continued)

\begin{tabular}{|c|c|c|c|}
\hline \multirow{2}{*}{$\begin{array}{l}\text { Characteristic } \\
\mathrm{CCl} \text { group, } \mathrm{n}(\%)^{\mathrm{b}}\end{array}$} & $\begin{array}{l}\text { Patients with } \\
\text { Major Bleeding } \\
\text { Events }(n=773)\end{array}$ & \multicolumn{2}{|c|}{$\begin{array}{l}\text { Patients with No } \\
\text { Major Bleeding } \\
\text { Events }^{\mathrm{a}}(\mathrm{n}=4,285)\end{array}$} \\
\hline & & & \\
\hline 0 & $(61.2)$ & 3,247 & $(75.8)$ \\
\hline 1 & $141 \quad(18.2)$ & 601 & $(14.0)$ \\
\hline 2 & $85 \quad(11.0)$ & 295 & $(6.9)$ \\
\hline$\geq 3$ & $(9.6)$ & 142 & (3.3) \\
\hline \multicolumn{4}{|l|}{ Comorbidities, n (\%) } \\
\hline Anemiab $^{\mathrm{b}}$ & $123 \quad(15.9)$ & 322 & $(7.5)$ \\
\hline Anxiety ${ }^{b}$ & $110 \quad(14.2)$ & 410 & $(9.6)$ \\
\hline Depression $^{b}$ & $129 \quad(16.7)$ & 451 & $(10.5)$ \\
\hline Fatigue $^{\mathrm{b}}$ & $169 \quad(21.9)$ & 581 & (13.6) \\
\hline Obesity & $43 \quad(5.6)$ & 247 & $(5.8)$ \\
\hline Thromboembolic events & $(7.5)$ & 110 & $(2.6)$ \\
\hline \multicolumn{4}{|l|}{ HCRU, n (\%) } \\
\hline Inpatient visits ${ }^{\mathrm{b}}$ & $171 \quad(22.1)$ & 619 & $(14.4)$ \\
\hline ED visits ${ }^{b}$ & $312 \quad(40.4)$ & 1,208 & $(28.2)$ \\
\hline Outpatient visits ${ }^{b}$ & $(99.4)$ & 4,204 & $(98.1)$ \\
\hline
\end{tabular}

${ }^{a}$ Each patient in the cohort with no major bleeding events was assigned an index date such that the index date distribution relative to the first diagnosis date of VWD matched that for the major bleeding events cohort.

${ }^{b}$ Variables that were significantly different between groups at baseline $(P<0.05$; Wilcoxon rank-sum test for continuous variables and chi-squared test or Fisher's exact test for categorical variables); these variables were controlled for as covariates in the post-index multivariate analysis.

Includes myocardial infarction, ischemic stroke, disseminated intravascular coagulation, deep vein thrombosis, pulmonary embolism, arterial embolism, acute but ill-defined cerebrovascular events, portal vein thrombosis, renal vein embolism and thrombosis, acute vascular insufficiency of intestine, retinal vascular occlusion, and vascular disorders of the kidney.

$\mathrm{CCl}=$ Charlson Comorbidity Index; $\mathrm{CDHP}=$ consumer-driven health plan; $E P O=$ exclusive provider organization; $E D=$ emergency department; $H C R U=$ health care resource utilization; $H D H P=$ high-deductible health plan; $P O S=$ point of service; $P P O=$ preferred provider organization; $V W D=$ von Willebrand disease.

Valid comparisons with prior prevalence data are difficult, owing to limited published data and differences in study methods and patient populations, especially given the rarity of the disease. A wide range of criteria have been used to define bleeding in patients with bleeding disorders, ${ }^{10}$ which can lead to considerable variation in prevalence estimates. In our sensitivity analysis, which excluded the requirement for transfusion of blood products in the case definition, the overall prevalence of major bleeding events nearly doubled compared with the primary analysis. Marked increases were observed in the prevalence estimates for menorrhagia, epistaxis, and joint bleeds. Notably, the overall prevalence of menorrhagia was more than 23 times greater in the sensitivity analysis than the primary analysis, suggesting that the requirement for transfusion may cause prevalence of menorrhagia to be underestimated. In a previous cross-sectional study, menorrhagia (defined as $\geq 2$ menorrhagia symptoms) occurred in $81 \%$ of women with VWD, but only $26 \%$ experienced menorrhagia requiring blood transfusion, factor VIII/VWF concentrate, desmopressin, or hysterectomy. ${ }^{11}$ For other bleed types, previous studies have reported prevalence rates of $18 \%$ for gastrointestinal bleeding and $23 \%$ for joint bleeding in patients with VWD. . $^{2,13}$

Although VWD is expected to affect men and women equally, women tend to seek medical care and may be diagnosed more often, owing to sex-specific hemostatic challenges (e.g., menorrhagia, postpartum hemorrhage) ${ }^{14}$ In the present analysis, female patients comprised three quarters $(75.2 \%)$ of the VWD cohort (with and without major bleeds), although most of the major bleeds observed during the study period were gastrointestinal-related rather than being specific to female patients. Furthermore, no obvious differences in types of major bleeds were observed between female and male patients (with the exception of menorrhagia).

To our knowledge, this is the first claims database study to report on the cost burden of bleeding events in patients with VWD. Patients who had major bleeding events experienced greater burden in terms of excess HCRU and direct health care costs than those without major bleeding events over the 12-month study period. Although the present study only evaluated direct health care costs, the significantly increased HCRU observed among patients with major bleeding events may suggest a higher burden of indirect costs owing to increased work/school absenteeism, loss of productivity, and decreased societal participation. The effect of major bleeding events in patients with VWD on these indirect costs is a topic for future research. The high level of overall health care and economic burden, and burden due to loss of productivity may also have a potential negative effect on the health-related quality of life of patients and caregivers.

The potential for serious hemorrhage during acute bleeding events ${ }^{15}$ requires effective hemostatic measures to restore functional levels of VWF and factor VIII; therefore, major bleeding events may bear significant clinical and economic implications. Unlike patients with hemophilia, however, many patients with VWD may not require regular replacement therapy. ${ }^{16}$ The National Heart, Lung, and Blood Institute guidelines recommend evaluating the risks and 


\section{FIGURE 3 Comparison of All-Cause HCRUa (OR by Health Care Visit) During the Study Period Between Patients with and Without Major Bleeding Events}

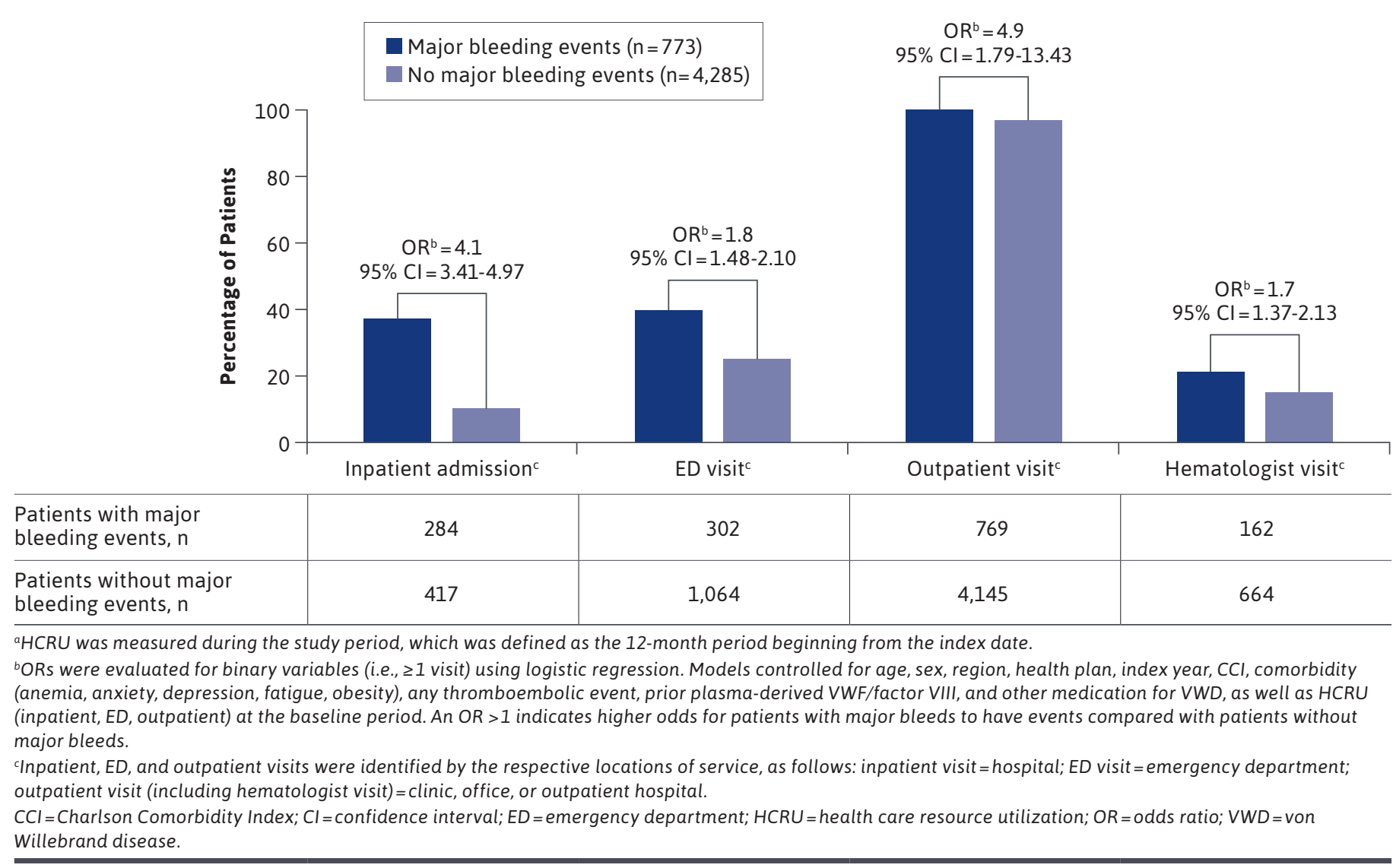

benefits of prophylaxis with VWF replacement therapies when considering long-term therapy for VWD. ${ }^{3}$

Prophylaxis use in patients with severe VWD has also been limited by a lack of awareness, the frequency of administration, perceived effectiveness, and cost among patients, caregivers, and health care providers. In addition, pharmacoeconomic studies evaluating the impact of prophylaxis in this population are lacking. Historically, there was a similar lack of data to support the use of prophylaxis in patients with severe hemophilia. ${ }^{17,18}$ Evidence from several randomized controlled studies has since confirmed the benefits of prophylaxis, which is now recognized as the standard of care for patients with severe hemophilia. ${ }^{18-20}$ Likewise, the benefits of prophylaxis have been shown in patients with severe VWD, ${ }^{6,7,15,21}$ with its potential to reduce long-term complications (e.g., joint damage) and to improve patients' overall quality of life.
Use of prophylaxis therapy to prevent or reduce the risk of major bleeding events also has the potential to reduce the health care and economic burden. Studies using data from the VWD Prophylaxis Network have reported the benefit of prophylaxis in patients with severe VWD to prevent nonmajor and major bleeds or reduce recurrent bleeding events. $6,7,15,21$ Abshire et al. (2013) reported a significant $(\mathrm{P}<0.0001)$ reduction in overall $\mathrm{ABR}$ among individuals with VWD on prophylaxis (vs. before prophylaxis) and in ABRs for specific bleed types, including epistaxis $(P=0.0005)$, joint bleeding $(P=0.002)$, and gastrointestinal bleeding $(P=0.001){ }^{21}$ Abshire et al. (2015) observed a decrease in median ABR from 25 before onset of prophylaxis to 6.1 during prophylaxis $(95 \%$ CI for rate difference, -51.6 to -1.7; $P=0.027$ ); at the final dosing level, median ABR was 4.0 $(95 \% \mathrm{CI}$ for rate difference, -57.5 to $-5.3 ; \mathrm{P}=0.0098) .{ }^{15} \mathrm{Holm}$ et al. (2015) found significant reductions in median ABRs for 


\section{FIGURE 4 Comparison of All-Cause Direct Health Care Costs During the Study Period Between Patients with and Without Major Bleeding Events}

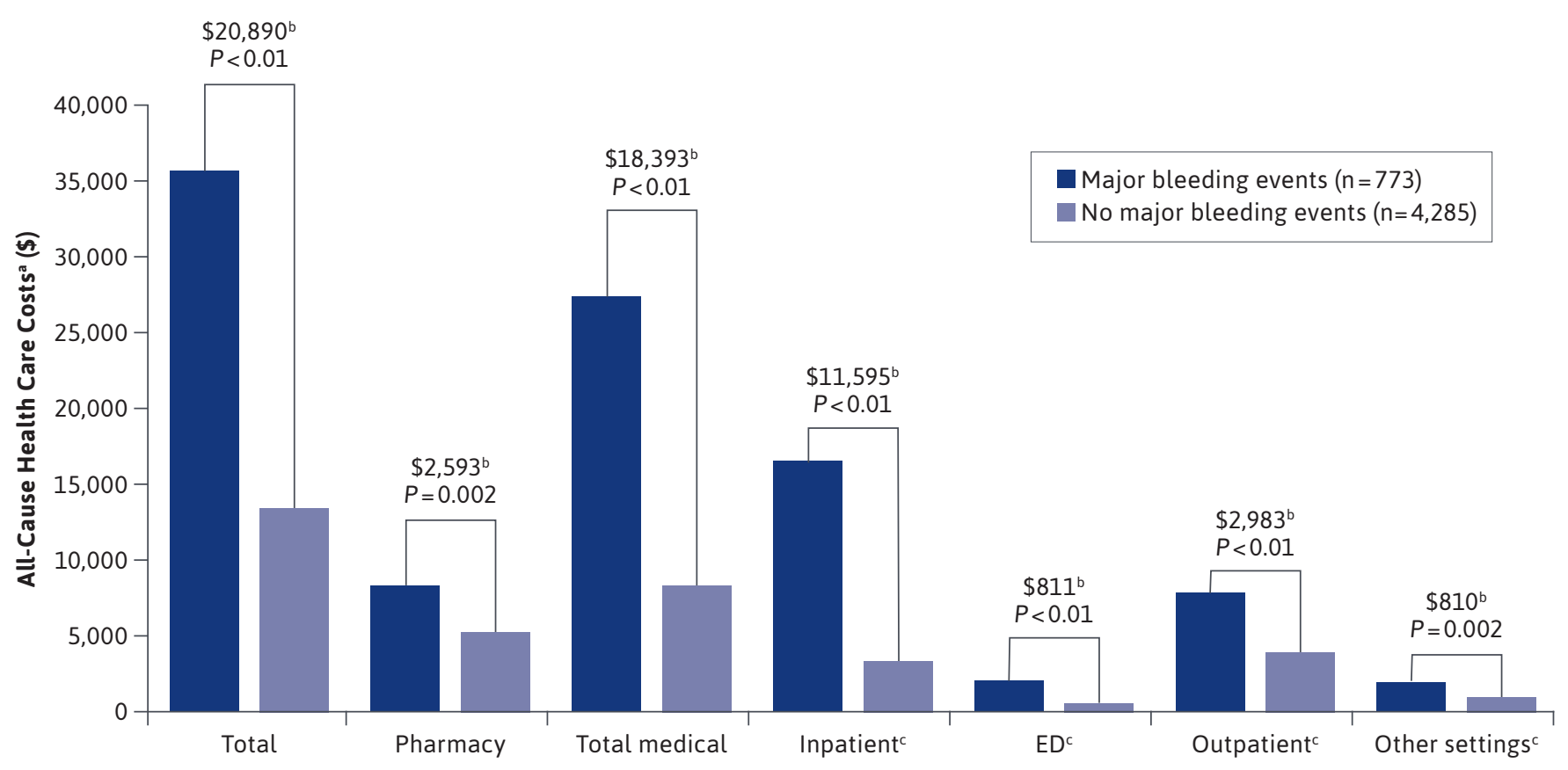

${ }^{a}$ Health care costs were measured during the study period, which was defined as the 12-month period beginning from the index date. All costs represent reimbursed amounts from payers to health care providers and were adjusted to 2016 U.S. dollars using the medical component of the Consumer Price Index. Total costs comprise pharmacy and total medical costs; total medical costs comprise inpatient, ED, outpatient, and other settings.

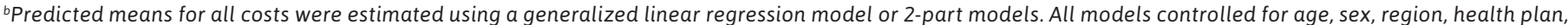

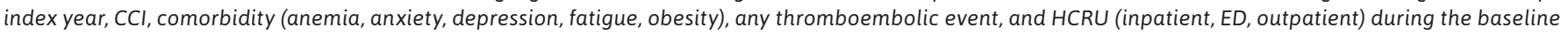

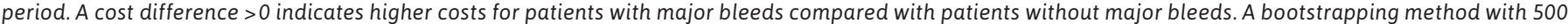
iterations was used to estimate the standard errors for the mean cost difference.

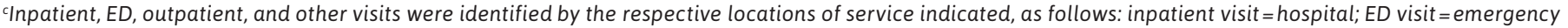
department; outpatient visit=clinic, office, or outpatient hospital; and other visit=home health care, hospice facility, inpatient rehabilitation, inpatient other, inpatient psychiatric, long-term care, outpatient rehabilitation, outpatient psychiatric, other location, other outpatient, pharmacy, skilled nursing facility, or surgical center.

$\mathrm{CCl}=$ Charlson Comorbidity Index; $\mathrm{ED}=$ emergency department; HCRU = health care resource utilization.

gastrointestinal bleeding (-44.3\%), epistaxis (-86.7\%), joint bleeding (-86.9\%), and menorrhagia (-100\%); patients with type 3 VWD had the most significant decrease in overall bleeding rate. ${ }^{6}$ In addition, a $40 \%$ reduction in the number of bleeding-related hospitalizations was observed following the initiation of prophylaxis $(\mathrm{P}=0.006)^{7}$

Type 3 VWD is generally considered to be severe, and patients may be placed on prophylaxis to prevent recurrent bleeding., ${ }^{1,22}$ Our study did not assess subtype classification and, therefore, the proportion of patients by VWD subtype is unknown. Assuming that patients with type 3 VWD in the dataset analyzed for this study are receiving prophylaxis, as recommended by treatment guidelines, the major bleeding rates observed in this analysis may reflect patients with type 1 or 2 VWD with a bleeding phenotype (i.e., severity) similar to patients with type 3 VWD not currently receiving prophylaxis. This highlights the need for patients to be managed on the basis of their bleed severity rather than their VWD subtype. To alleviate disease burden and significantly reduce HCRU, prophylaxis may need to be adopted as standard of care in all patients with severe VWD experiencing major bleeds, irrespective of their VWD type.

\section{LIMITATIONS}

This analysis has several limitations. Study results may not be generalizable beyond the study population, as data used in this analysis were limited to patients within a U.S. commercial health care plan. Claims data also may lack 
specific clinical information, which may limit the accurate identification of cases of VWD and major bleeding events. In another analysis by Sidonio et al. (2017), which also utilized ICD9 -CM codes ( $\geq 2$ claims for VWD) to identify patients with VWD, less than two thirds of patients had a diagnostic laboratory test within the 2 years before or after diagnosis. ${ }^{23}$ The Sidonio analysis, therefore, raises questions concerning the reliability of using ICD-9-CM claims data alone to identify patients with VWD. The analysis reported herein included criteria (e.g., exclusion of laboratory and radiology claims) to minimize false positives. However, this approach may not have completely eliminated this issue. Another potential approach would have been to combine diagnostic codes with treatment and/or procedural codes, as used in a previous cost analysis in patients with hemophilia. ${ }^{24}$

As noted above, definitions of major bleeding events vary across the literature. ${ }^{10}$ We utilized a conservative definition based on the ISTH definition created for patients receiving antihemostatic medical products. ${ }^{8}$ The sensitivity analysis suggests that the use of transfusion with blood products in an outpatient setting to define major bleeding events may have resulted in an underestimation of the prevalence of major bleeding events in the primary analysis. Patients with VWD who experience major bleeding events (e.g., menorrhagia, epistaxis, and joint bleeds) may not necessarily require transfusion but may nevertheless suffer from excessive bleeding necessitating medical care. However, the inclusion of such patients in the HCRU and cost analysis would have likely resulted in an underestimation of the overall costs and HCRU, although the relationship between HCRU and severity of major bleeds has yet to be determined. Patients with major joint bleeds may have also been receiving home therapy and thus may have been missed by both the requirement for transfusions in the primary analysis and laboratory tests within 7 days of the bleeding event in the sensitivity analysis.

\section{Conclusions}

Although our estimate of the prevalence of major bleeding events may be conservative, this is the first study to use a large dataset to evaluate the health care and economic burden associated with major bleeding events in patients with VWD. Major bleeding events were associated with high HCRU and costs (driven by inpatient costs); therefore, optimizing therapy for patients with VWD to prevent or reduce the risk of major bleeding events is critical for reducing health care and economic burden.

\section{DISCLOSURES}

This study was funded by Baxalta U.S. Inc., a Takeda company (Lexington, MA). The study sponsor was involved with the study design, analysis, and interpretation of data; writing of the manuscript; and the decision to publish the article. $\mathrm{Lu}, \mathrm{Wu}$, and Ewenstein are employees of Baxalta U.S. Inc., a Takeda company, and are Takeda stock owners. Farahbakhshian is an employee of Shire U.S. Inc., a Takeda company, and is a Takeda stock owner. Oladapo was an employee of Baxalta U.S. Inc., a Takeda company, at the time the analysis was completed and the manuscript developed, and is a Takeda stock owner.

\section{ACKNOWLEDGMENTS}

Under the direction of the authors, medical writing support for this manuscript was provided by Joanne Vaughan, BSc, employee of Excel Medical Affairs (Fairfield, CT) and was funded by Baxalta U.S. Inc., a Takeda company (Lexington, MA).

\section{REFERENCES}

1. Leebeek FW, Eikenboom JC. Von Willebrand's disease. N Engl J Med. 2016;375(21):2067-80.

2. Bowman M, Hopman WM, Rapson D, Lillicrap D, James P. The prevalence of symptomatic von Willebrand disease in primary care practice. J Thromb Haemost. 2010;8(1):213-16.

3. Nichols WL, Hultin MB, James AH, et al. Von Willebrand disease (VWD): evidence-based diagnosis and management guidelines, the National Heart, Lung, and Blood Institute (NHLBI) Expert Panel report (USA). Haemophilia. 2008;14(2):171-232.

4. Stockschlaeder M, Schneppenheim R, Budde U. Update on von Willebrand factor multimers: focus on high-molecular-weight multimers and their role in hemostasis. Blood Coagul Fibrinolysis. 2014;25(3):206-16.

5. Sadler JE, Budde U, Eikenboom JC, et al. Update on the pathophysiology and classification of von Willebrand disease: a report of the subcommittee on von Willebrand Factor. J Thromb Haemost. 2006;4(10):2103-14.

6. Holm E, Abshire TC, Bowen J, et al. Changes in bleeding patterns in von Willebrand disease after institution of long-term replacement therapy: results from the von Willebrand Disease Prophylaxis Network. Blood Coagul Fibrinolysis. 2015;26(4):383-88.

7. Holm E, Carlsson KS, Lövdahl S, Lail AE, Abshire TC, Berntorp E. Bleeding-related hospitalization in patients with von Willebrand disease and the impact of prophylaxis: results from national registers in Sweden compared with normal controls and participants in the von Willebrand Disease Prophylaxis Network. Haemophilia. 2018;24(4):628-33. 
8. Schulman S, Kearon C; Subcommittee on Control of Anticoagulation of the Scientific and Standardization Committee of the International Society on Thrombosis and Haemostasis. Definition of major bleeding in clinical investigations of antihemostatic medicinal products in non-surgical patients. J Thromb Haemost. 2005;3(4):692-94.

9. Riley L, Womack M, Zappa S. Emergency room care. Accessed May 19, 2020. https:// www.hemophilia.org/sites/default/files/ document/files/nurses-guide-chapter8-emergency-room-care.pdf

10. Tosetto A, Castaman G, Rodeghiero F. Bleeders, bleeding rates, and bleeding score. J Thromb Haemost. 2013;11(Suppl 1): 142-50.

11. De Wee EM, Knol HM, MauserBunschoten EP, et al. Gynaecological and obstetric bleeding in moderate and severe von Willebrand disease. Thromb Haemost. 2011;106(5):885-92.

12. Castaman G, Federici AB, Tosetto A, et al. Different bleeding risk in type 2A and $2 \mathrm{M}$ von Willebrand disease: a 2-year prospective study in 107 patients. $\mathrm{J}$ Thromb Haemost. 2012;10(4):632-38.
13. van Galen KP, Sanders YV, Vojinovic U, et al. Joint bleeds in von Willebrand disease patients have significant impact on quality of life and joint integrity: a cross-sectional study. Haemophilia. 2015;21(3):e185-92.

14. James AH. Von Willebrand disease in women: awareness and diagnosis. Thromb Res. 2009;124(Suppl 1):S7-10.

15. Abshire T, Cox-Gill J, Kempton CL, et al. Prophylaxis escalation in severe von Willebrand disease: a prospective study from the von Willebrand Disease Prophylaxis Network. J Thromb Haemost. 2015;13(9):1585-89.

16. Castaman G, Linari S. Diagnosis and treatment of von Willebrand disease and rare bleeding disorders. J Clin Med. 2017;6(4):pii:E45.

17. Iorio A, Marchesini E, Marcucci M, Stobart K, Chan AK. Clotting factor concentrates given to prevent bleeding and bleeding-related complications in people with hemophilia A or B. Cochrane Database Syst Rev. 2011;(9):CD003429.

18. Saccullo G, Makris M. Prophylaxis in von Willebrand disease: coming of age? Semin Thromb Hemost. 2016;42(5):498-506.
19. Gringeri A, Lundin B, von Mackensen S, Mantovani L, Mannucci PM. A randomized clinical trial of prophylaxis in children with hemophilia A (the ESPRIT study). J Thromb Haemost. 2011;9(4):700-10.

20. Manco-Johnson MJ, Abshire TC, Shapiro AD, et al. Prophylaxis versus episodic treatment to prevent joint disease in boys with severe hemophilia. N Engl J Med. 2007;357(6):535-44.

21. Abshire TC, Federici AB, Alvárez MT, et al. Prophylaxis in severe forms of von Willebrand's disease: results from the von Willebrand Disease Prophylaxis Network (VWD PN). Haemophilia. 2013;19(1):76-81.

22. Neff AT, Sidonio RF, Jr. Management of VWD. Hematology Am Soc Hematol Educ Program. 2014;2014(1):536-41.

23. Sidonio RF, Haley KM, Fallaize D. Impact of diagnosis of von Willebrand disease on patient outcomes: analysis of medical insurance claims data. Haemophilia. 2017;23(5):743-49.

24. Armstrong EP, Malone DC, Krishnan S, Wessler MJ. Costs and utilization of hemophilia A and B patients with and without inhibitors. J Med Econ. 2014;17(11):798-802. 